\title{
Haptic form perception: Relative salience of local and global features
}

\author{
STEPHEN LAKATOS and LAWRENCE E. MARKS \\ John B. Pierce Laboratory and Yale University, New Haven, Connecticut
}

\begin{abstract}
When we examine objects haptically, do we weight their local and global features as we do visually, or do we place relatively greater emphasis on local shape? In Experiment 1, subjects made either haptic or visual comparisons of pairs of geometric objects (from a set of 16) differing in local and global shape. Relative to other objects, those with comparable global shape but different local features were judged less similar by touch than by vision. Separate groups of subjects explored the same objects while wearing either thick gloves (to discourage contour-following) or splinted gloves (to prevent enclosure). Ratings of similarity were comparable in these two conditions, suggesting that neither exploratory procedure was necessary, by itself, for the extraction of either local or global shape. In Experiment 2, haptic exploration time was restricted to $1,4,8$, or 16 sec. Limiting exploration time affected relative similarity in objects differing in their local but not their global shape. Together, the findings indicate that the haptic system initially weights local features more heavily than global ones, that this differential weighting decreases over time, and that neither contour-following nor enclosure is exclusively associated with the differential emphasis on local versus global features.
\end{abstract}

In recent years, considerable effort in haptic research has been directed toward characterizing spatial processing capabilities. The haptic system combines both cutaneous and kinesthetic information to construct an internal representation of external objects and spatial configurations, using both bottom-up processes that convey topographical input concerning spatial layouts and top-down processes that optimize search procedures directed at specific types of spatial features. Two theoretical perspectives can be discerned in the literature: The first compares haptic with visual spatial processing, seeking perceptual equivalences (or nonequivalences) between touch and vision (e.g., Brown \& Brumaghim, 1968; Garbin \& Bernstein, 1984); the second highlights the role of nonspatial cues (e.g., texture, hardness, temperature) and task-driven exploratory procedures, such as prototypical hand movements, in haptic form perception, assuming the existence of separate processing channels for touch and vision (e.g., Klatzky \& Lederman, 1993).

Several researchers have reported a strong equivalence between visual and haptic form perception. Brown and Brumaghim (1968) found that haptic and visual ratings of complexity of stimuli drawn from a set of 1,000 2-D

This research was supported in part by National Research Service Fellowship Award F32 DC00174 from the National Institutes of Health to S.L. and Grant DC00271-13 from the National Institutes of Health to L.E.M. We thank Erica Boiman and Neela Moorty for their assistance in running subjects in Experiment 1. Correspondence should be addressed to S. Lakatos, Department of Psychology, Washington State University, 14204 N.E. Salmon Creek Avenue, Vancouver, WA 98686 (e-mail: lakatos@vancouver.wsu.edu).

-Accepted by previous editor, Myron L. Braunstein polygons (see Brown \& Owen, 1967) were highly similar and were linearly related to the number of independent sides; visual and haptic ratings of the size of these stimuli were also moderately correlated (Owen \& Brown, 1970). More recently, Garbin and Bernstein (1984) had subjects sort 24 nonrepresentative forms either visually or haptically according to similarity of shape and obtained comparable 2-D multidimensional scaling (MDS) solutions for which the principal dimensions were size and shape. Such visual-tactile perceptual equivalence appears to be even more prominent in children than in adults (Garbin, 1990). Taken together, these findings suggest that touch and vision may share common processing mechanisms or, as Gibson (1966) noted, that "touch and vision in combination often yield a redundant, doubly guaranteed input of information" (p. 53).

Haptic touch nonetheless can produce substantial errors and distortions in certain types of spatial tasks, especially tasks involving the recognition of raised-line depictions of 2-D and 3-D images (Magee \& Kennedy, 1980) and the perception of the spatial properties of simple point and line stimuli (Balakrishnan, Klatzky, Loomis, \& Lederman, 1989). Testing haptic recognition with highly prototypical 2-D displays of common objects (e.g., a hammer), Lederman, Klatzky, Chataway, and Summers (1990) reported high error rates $(34 \%$ correct) and lengthy exploration times $(91 \mathrm{sec})$. Congenitally blind subjects, who presumably would have more extensive perceptual resources to bring to bear on such tasks, performed even worse ( $10 \%$ correct). By contrast, vision is extremely fast and accurate in such contexts. This outcome is paradoxical, and is especially puzzling in light of Klatzky, Lederman, and Metzger's (1985) finding that people can be exceedingly quick and accurate in recog- 
nizing by touch the actual (3-D) objects themselves. In these experiments, when asked to identify 100 common objects by touch alone, subjects typically responded within $2 \mathrm{sec}$ and with $100 \%$ accuracy. Such apparent discrepancies in the ability of the haptic system to assimilate and interpret different kinds of spatial information suggest that haptic representation of spatial information is not wholly equivalent to that of vision, and that the spatial integration mechanisms of touch may pose modality-specific constraints.

Thus, under most circumstances, haptic recognition of objects does exceed recognition of 2-D representations of objects (although see Heller, Calcaterra, Burson, \& Tyler, 1996, for instances in which 2-D pictures may yield high levels of accuracy). Further, it appears that the choice of stimuli-2-D or 3-D - may itself relate to implicit assumptions regarding how the haptic system processes information. Lederman and Klatzky (1987) distinguished between "image-mediation" and "direct apprehension" models of haptic processing. According to the image-mediation model, spatially coarse, temporally extended haptic information is translated into a visual image and is then interpreted and represented visually. From this perspective, haptic processing is viewed as an inferior form of vision. By contrast, according to the direct apprehension model, touch possesses spatial processing mechanisms that are physiologically distinct from those of vision. Only at later stages are visual and haptic representations available for shared processing. In this model, touch has access to structural properties of objects (e.g., shape, size), as well as what Lederman et al. (1990) referred to as "substance" properties (e.g., surface texture, hardness, and thermal characteristics). Highly precise spatial (structural) discriminations of form are therefore unnecessary, because the haptic system can recognize natural, common objects by deriving information through both approximate spatial heuristics and nonspatial (substance) cues.

Exploratory hand movements represent an integral component of the direct haptic apprehension model, because each of these movements is optimized to detect information about a particular stimulus property. Two principal exploratory procedures are used for judging the shape of an object. The first is enclosure, in which the fingers are molded to an object's contours in order to obtain a general impression of global shape and volume. Enclosure is often followed by contour-following to interpret the small-scale features of shape, as well as to confirm aspects of global shape derived from enclosure. When classifying common objects at the basic and subordinate level (see Rosch, Mervis, Gray, Johnson, \& Boyes-Braem, 1976), people tend to use a two-stage sequence, consisting of an initial grasp-and-lift procedure (i.e., enclosure, accompanied by unsupported holding), to extract basic-level shape characteristics. This initial step is followed by more specialized exploratory movements (e.g., contour-following for exact shape, lateral motion for texture) to perceive details of subordinate spatial and nonspatial properties (Lederman \& Klatzky,
1990). Thus, touch has a considerable repertoire of specialized hand movements available to evaluate both local and global aspects of object shape.

The present experiments sought to build upon previous work by evaluating how the haptic system processes small- and large-scale features of object shape. Specifically, when we explore one or more objects haptically, as opposed to visually, what relative emphasis do we place on local and global shape? By "local features," we refer to a subordinate unit of macrostructure rather than to the notion of microstructure (i.e., texture) as defined by Katz (1989).

Studies of categorization (Rosch et al., 1976; Tversky \& Hemenway, 1984) have shown that objects tend to be organized at the "basic" level, so that objects within a single category maximize the number of attributes they have in common, whereas objects in different categories minimize shared attributes. Furthermore, basic-level distinctions are typically made on the basis of part structure, while those at more specific (subordinate) classification levels tend to share parts. There is no a priori reason, however, why different modalities such as touch and vision should necessarily place equivalent emphasis on either the basic-level part structure or subordinate features of an object. Vision, through a 2-D retinal image, has simultaneous access to both local and global features of an object. Touch, on the other hand, engages in a series of temporally extended exploratory procedures, each of which typically scans a portion of the potentially available shape cues. Although there are instances in which individuals may execute only an enclosure when they wish to obtain coarse information about global shape and volume, as well as other object dimensions (see, e.g., Klatzky \& Lederman, 1995), and although enclosure is "sufficient" to produce above-chance performance with respect to matching objects on dimensions other than global shape, only contour-following is "optimal" for extracting local shape information.

Given that touch typically constructs a detailed impression of object shape through successive sweeps of relatively small surface areas, we predicted that touch would weight small-scale, local features more heavily than would vision. It is important to note, however, that scale is relative and it is difficult to know how to define small- or large-scale space for the finger, hand, or arms (Millar, 1991); hence, our use of the terms local and global to describe our stimuli does not refer to any absolute scale for size, but rather reflects those spatial properties of the stimuli that differentiate them at subordinated and basic levels, respectively.

In Experiment 1, we obtained haptic and visual ratings of similarity using $163-\mathrm{D}$ wooden objects. The objects were abstract geometric shapes of low to moderate levels of complexity. They were selected to provide eight primary pairs, each of which shared characteristics of global shape but differed in their local features. Thus, the eight pairs of objects varied in both local and global shape. We were interested primarily in similarity ratings given to the eight primary pairs: Would touch and vision 
assign equivalent similarity ratings to them (relative to the overall mean rating for each modality), or, as we predicted, would touch judge these pairs to be relatively less similar than vision?

We also predicted that haptic weighting of local and global shape characteristics would depend upon the repertoire of exploratory hand movements that could be applied optimally in the comparison task. Therefore, in two additional conditions of Experiment 1, we restricted subjects' hand movements by having the subjects wear either thick rubber gloves, which discouraged extensive contour-following, or splinted gloves, which prevented flexing of fingers and enclosing an object. Subjects in the thick-glove condition were instructed to use enclosure exclusively in their exploration, while those wearing splinted gloves were instructed to engage only in contour-following. On the basis of previous findings, we predicted that the subjects wearing thick gloves and engaging in enclosure would tend to weight global shape more heavily than bare-handed subjects, whereas subjects wearing splinted gloves and employing contourfollowing movements would rely primarily on impressions of local features. If, on the other hand, similarity ratings did not differ across conditions, then one could conclude that each exploratory movement by itself provides sufficient information to judge both local and global shape in the present stimulus set, but that neither is essential.

Finally, we examined how limiting the time for exploration might affect haptic similarity ratings of shape. Because hand movements are necessarily extended in time, we expected some interaction between exploration time and judgments of haptic shape. Increasing haptic exploration time has been shown to improve matching performance (Davidson, Abbott, \& Gershenfeld, 1974), suggesting that a haptic representation of object shape is constructed over time. Subjects in Experiment 2 compared selected pairs of the 16 geometric objects over observation intervals ranging from 1 to $16 \mathrm{sec}$. At short intervals, we anticipated that subjects could obtain only a rough estimate of general shape through enclosure. With longer time intervals, subjects should be able to deploy a more comprehensive array of exploratory procedures, including contour-following, and consequently obtain a more accurate impression of object shape. With the present stimulus set, we tested whether ratings of similarity of pairs of objects differing only in local features would depend strongly on exploration time, because such features would normally be extracted through extensive and detailed hand movements. Perception of objects differing primarily in global shape, however, should depend less on exploration time because enclosure can be performed quite rapidly and is diagnostic of general overall shape.

\section{EXPERIMENT 1}

\section{Method}

Subjects. One hundred twelve individuals ( 79 women, 34 men) between the ages of 18 and 30 were recruited through posters on the
Yale University campus and reimbursed for their participation. Subjects were divided among four conditions: (1) Nineteen women and 10 men made haptic comparisons (Condition 1); (2) 18 women and 9 men made visual comparisons (Condition 2); (3) 21 women and 7 men made haptic comparisons while wearing thick gloves (Condition 3); and (4) the remaining 28 subjects ( 20 women, 8 men) compared stimuli while wearing splinted gloves (Condition 4).

Stimuli. Sixteen stimuli were selected from a collection of precision-cut wooden objects encompassing a wide variety of abstract geometric shapes (Wards Scientific). These objects are typically used to instruct geology students about the structural characteristics of crystal shapes. Their dimensions $\left(100-200 \mathrm{~cm}^{3}\right)$ are small enough so that each object can easily be grasped and enclosed with either one or both hands. For the present study, objects were selected to form eight primary pairs in such a way that the members of each pair shared roughly similar characteristics of global shape, but differed in terms of the presence or absence of local features (i.e., despite sharing global shape, one of the two members possessed small-scale features). For the members of certain pairs, the presence of local features (e.g., grooves along the intersections of adjacent sides) did not substantially alter their global shape. For other pairs, however, local transformations of shape were more complex and interacted with global shape, to the point where only a subset of global shape cues remained intact. Both global and local shape varied across pairs of objects.

Table 1 outlines the general shape characteristics of the stimuli. Each of the 16 objects can be classified in general geometric terms as a tetragon, hexagon, octagon, or a combination of a dodecahedron and a trapezohedron. Three general types of tetragons were used: a cube (Object 1A), a smaller rhomboid shape with nonperpendicular sides (Object $2 \mathrm{~A}$ ), and a larger rectangular column (Object 3A). Object $1 \mathrm{~B}$ shares the overall cubical dimensions of Object 1A, but represents a complex transformation comprising two tritetrahedra with parallel axes. Similarly, Object $2 B$ possesses the same overall dimensions as Object $2 \mathrm{~A}$, except that complex concave surfaces replace flat rectangular sides. Object $3 \mathrm{~B}$ is identical to $\mathrm{Ob}$ ject $3 \mathrm{~A}$, except that seven small triangular spikes have been distributed across four of its sides.

Among the objects with hexagonal geometries, Objects $5 \mathrm{~A}$ and $5 \mathrm{~B}$ are both di-pyramidal hexagons, but the former has six symmetrical occlusions spanning pairs of sides. Objects $6 \mathrm{~A}$ and $6 \mathrm{~B}$ are both hexagonal columns with identical dimensions, except that Object $6 \mathrm{~B}$ has shallow grooves ( $3 \mathrm{~mm}$ wide) running the length of four of its sides. Similarly, Objects $7 \mathrm{~A}$ and $7 \mathrm{~B}$ are distinguished only by the latter's two depressions at the center of its two largest sides, giving this object a "doughnut-like" appearance. For both the octagonal pair (Objects 4A and 4B) and the dodecahedral/trapezohedral pair (Objects $8 \mathrm{~A}$ and $8 \mathrm{~B}$ ), distinguishing surface features consist of 3-mm-wide grooves running along the intersections of all pairs of adjacent sides.

Apparatus and Procedure. All subjects were tested individually in an isolated room. On each trial of Condition 1, subjects were presented simultaneously with a pair of objects behind an opaque curtain that permitted them to feel the objects, but not to see them, and were instructed to explore the objects for approximately $15 \mathrm{sec}$. No restrictions were placed on the types of hand movement or exploratory procedures that subjects could use. Throughout Experiments 1 and 2, subjects routinely initiated explorations with both hands simultaneously, touching one member of a pair with the left hand while at the same time touching the other member of the pair with the right hand.

After this period of free exploration, subjects rated the overall degree of similarity between the shapes of the two objects by adjusting the position of a continuous sliding switch of a similarity scale presented on the screen of a NeXT microcomputer. No a priori definition of "shape" was provided. The left and right endpoints of the scale were labeled "very similar" and "very different," respectively. The scale comprised approximately 500 discrete positions between these endpoints. Subjects were instructed to use the full range of the 
Table 1

Geometric Classification of the 16 Haptic Shapes Used in Experiments 1-3

\begin{tabular}{|c|c|c|c|}
\hline Object No. & $\begin{array}{l}\text { General } \\
\text { Geometry }\end{array}$ & $\begin{array}{l}\text { Global } \\
\text { Shape }\end{array}$ & $\begin{array}{l}\text { Additional Local } \\
\text { Features }\end{array}$ \\
\hline $1 \mathrm{~A}$ & Tetragon & Cube & None \\
\hline 1B & $"$ & $"$ & $\begin{array}{l}\text { Complex transformation: two tritetrahedra } \\
\text { with parallel axes }\end{array}$ \\
\hline $2 A$ & $"$ & Rhombus & None \\
\hline $2 B$ & $"$ & $"$ & $\begin{array}{l}\text { Seven small triangular spikes } \\
\text { distributed across four sides }\end{array}$ \\
\hline $3 \mathrm{~A}$ & $"$ & Column & None \\
\hline $3 B$ & $"$ & $"$ & $\begin{array}{l}\text { Complex transformation: concave } \\
\text { surfaces replace rectangular sides }\end{array}$ \\
\hline $4 \mathrm{~A}$ & Octagon & Di-pyramid & None \\
\hline $4 B$ & $"$ & $"$ & $\begin{array}{l}\text { Moderate grooves at intersections } \\
\text { between sides }\end{array}$ \\
\hline $5 \mathrm{~A}$ & Hexagon & Di-pyramid & None \\
\hline $5 B$ & $"$ & $"$ & $\begin{array}{l}\text { Deep grooves/occlusions along pairs } \\
\text { of sides (four large, two small) }\end{array}$ \\
\hline $6 \mathrm{~A}$ & $"$ & Column & None \\
\hline $6 \mathrm{~B}$ & $"$ & $"$ & $\begin{array}{l}\text { Shallow grooves running along } \\
\text { length of column (four sides) }\end{array}$ \\
\hline $7 \mathrm{~A}$ & $"$ & $\begin{array}{l}\text { Truncated } \\
\text { di-pyramid }\end{array}$ & None \\
\hline $7 \mathrm{~B}$ & $"$ & $"$ & $\begin{array}{l}\text { Two hexagonal depressions at center } \\
\text { of two largest opposing sides }\end{array}$ \\
\hline $8 \mathrm{~A}$ & $\begin{array}{l}\text { Dodecahedron/ } \\
\text { trapezohedron }\end{array}$ & Spherical & None \\
\hline $8 \mathrm{~B}$ & $"$ & $"$ & $\begin{array}{l}\text { Shallow grooves at intersections } \\
\text { between adjacent sides }\end{array}$ \\
\hline
\end{tabular}

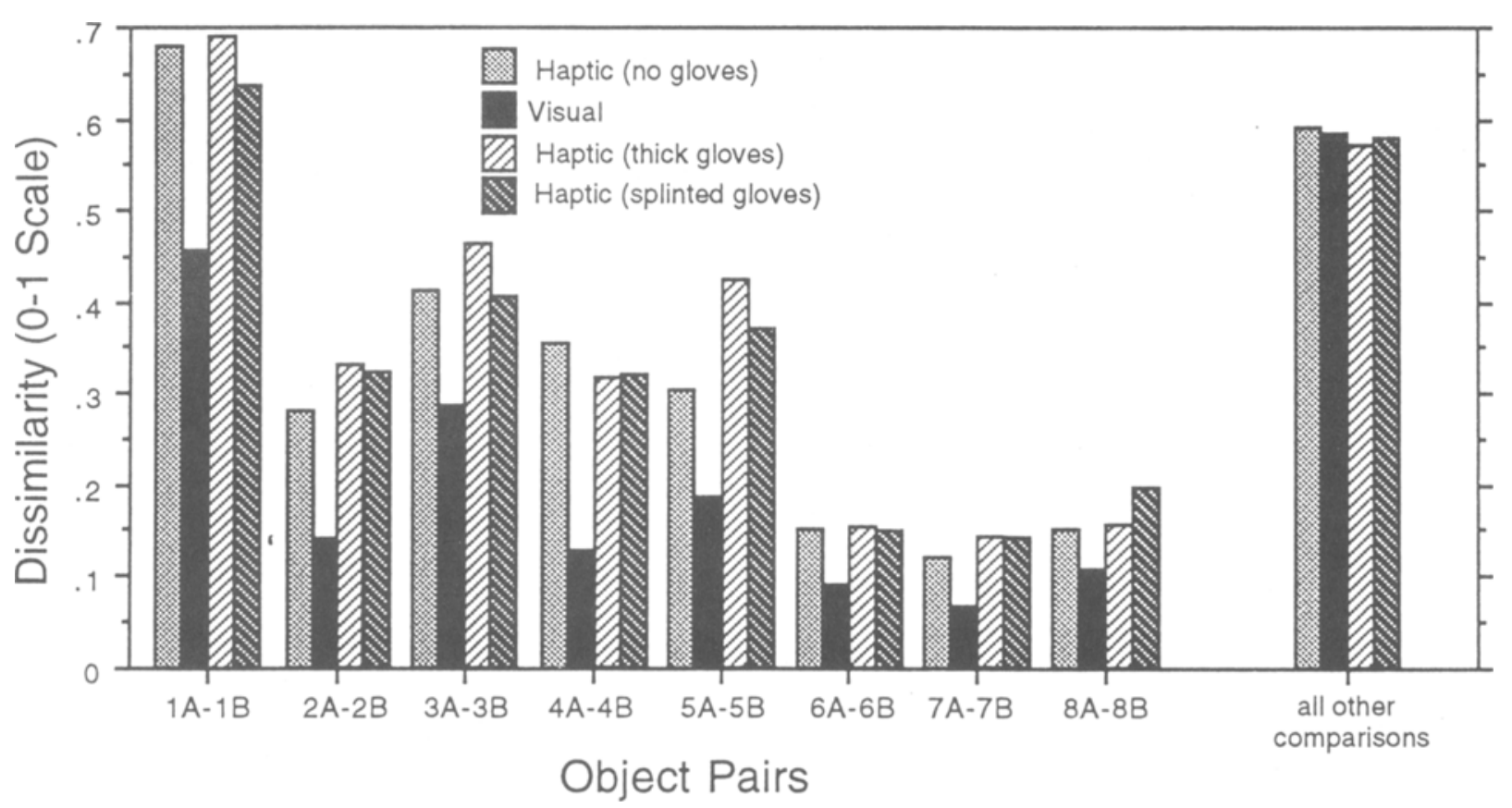

Figure 1. Average dissimilarity ratings from Experiment 1 for the eight object pairs with similar global shape, but differing local structure, are shown separately for each of the four conditions. Pooled ratings for all other comparisons within the four conditions are shown at the right. 
similarity scale and to distribute their responses as uniformly as possible, but were cautioned against moving the sliding switch to the extreme right (i.e., "very different") side of the scale on the initial trials in order to allow room for potentially more different pairs later on.

Subjects in Condition 2 (visual) followed a procedure similar to that of Condition 1 , except that they viewed the shapes as they were presented on a matte white surface by the experimenter. In order to encourage subjects to consider shape from a variety of visual perspectives, and to make visual exploration more closely resemble that of touch, the experimenter rotated both objects along a number of different axes and in a number of different orientations during the presentation. Distractions caused by the experimenter partially occluding portions as he/she held it for the observer were also minimized by this procedure. Subjects were also told that no orientation of either object should be given special preference over another.

Subjects in Condition 3 (thick gloves) explored the stimuli wearing heavy rubber gloves with a thickness of $30 \mathrm{mil}$ (.030 in.) at each fingertip. The gloves were designed for heavy cleaning conditions (e.g., corrosive solvents) and were sufficiently thick to attenuate many of the local stimulus features. Subjects in this condition were instructed to use only enclosure during exploration of the objects; the experimenter demonstrated this procedure to subjects before the session and monitored subjects' hand movements during each trial in order to ensure proper technique. Subjects in the splinted-glove condition (Condition 4) donned woolen gloves into which 1-mm stainless steel rods - running along the back of each finger-had been woven in order to prevent flexure of the digits. The woolen gloves had exposed fingertips to encourage subjects to employ contour-following, but not enclosure, as an exploratory procedure. Small, medium, and large versions of both the rubber and splinted gloves were available to subjects in order to accommodate differences in hand size. Subjects in Condition 4 were also instructed to avoid enclosing the objects and to use contour-following exclusively during exploration. In all other respects, the procedures across the four conditions were identical.

Before the start of either condition, subjects were provided with a familiarization session in which they explored each of the 16 shapes for approximately $20 \mathrm{sec}$. Subjects in Conditions 3 and 4 wore their gloves for this session. The purpose of this initial phase was to provide subjects with an idea of the range and variability of shapes in the stimulus set so that they would have some notion of how the endpoints of the similarity scale might be anchored. After the familiarization sequence, subjects completed 240 trials, spread across two $1-h$ sessions on consecutive days (one complete presentation of the pairs in each session). The trials comprised each possible pairing of objects presented in a randomized order. Presentation of stimulus pairs was counterbalanced across hands, so that both members of each pair were presented to both the left and the right hands within a session.

\section{Results}

Ratings of dissimilarity for each pair of objects were averaged separately across subjects in each of the four conditions to yield four $16 \times 16$ dissimilarity matrices. Figure 1 compares dissimilarity ratings for the eight pairs of objects with similar global shape but differing local structure (averaged ratings for all other paired comparisons are combined and shown at the right of the figure) in the four conditions. Relative to subjects in Condition 1 , subjects in the visual condition rated the eight pairs of objects as more similar, relative to the average rating for all other stimulus pairs. That is, the shapes of these 8 pairs felt relatively more alike visually than haptically, regardless of the constraints of haptic exploration. In carrying out a repeated measures analysis of variance (ANOVA), we grouped data in both Condi- tions 1 and 2 into two categories - one representing the eight pairs of objects for which only local shape varied and the other representing the remaining 112 pairs for which global shape (and possibly local shape) variedthus yielding a nested design with condition as the between-subjects variable and stimulus category as the within-subjects variable. ANOVA tests indicated a significant main effect of condition $[F(1,54)=19.49, p<$ $.001]$ and of category $[F(1,54)=1,061.26, p<.001]$, as well as a significant interaction $[F(1,54)=38.46, p<$ $.001]$ between these variables. Although we did not carry out formal analyses of subjects' exploratory movements, our informal observations indicated that subjects typically engaged in the two-stage exploration sequence described by Lederman and Klatzky (1990), in which they first executed enclosure in order to gain a coarse sense of shape and subsequent contour-following in order to extract more precise spatial information. At the end of each exploration period for a stimulus pair, most subjects tended to enclose both objects one last time, as if to corroborate with this technique the spatial impressions they had obtained previously through contour-following. We also noted somewhat more extended contour-following for those instances in which the pair of objects shared similar global shape but different local features.

From Figure 1, it is also apparent that, contrary to our expectations, the introduction of different constraints on the repertoire of exploratory procedures available to subjects had little effect on their dissimilarity judgments relative to the overall means for all other paired comparisons. An ANOVA for Conditions 3 and 4 revealed no significant main effect of condition $[F(1,54)=.059, p=$ $.81]$, a significant effect of category $[F(1,54)=353.05$, $p<.001]$, and no significant interaction between these variables $[F(1,54)=1.38, p=.25]$. The nonsignificant interaction implies that subjects in both gloved conditions gave more or less equivalent similarity ratings to the eight pairs of objects possessing similar global characteristics, but different local features, relative to the mean ratings for all other pairs - or, more conservatively, that donning thick versus splinted gloves failed to produce a reliable difference in processing local versus global features. Subjects apparently judged the similarity of shape on the basis of sensory input that was available through either contour-following or enclosure. This finding is unexpected in light of past research showing that exploratory movements are optimized to extract distinct types of spatial information and suggests that, under certain conditions, the haptic system can successfully interpret spatial parameters obtained through nonoptimal hand movements.

In order to visualize the proximity relations among stimuli within each condition, we obtained clustering solutions by submitting the dissimilarity matrices to the EXTREE computer program (Corter \& Tversky, 1986). The algorithm used by EXTREE is an extension of the additive feature tree (see Sattath \& Tversky, 1977), which relaxes the ultrametric inequality assumption in hierarchical clustering models (i.e., any 3 points can be 
labeled $x, y, z$ such that $d[x, z]=d[y, z] \geqslant d[x, y]$ ) in favor of a model of similarity in which the leaves of the tree are not necessarily equidistant from the root.

The EXTREE algorithm provides an additional feature for detecting not only nested, but also overlapping, clusters within proximity data by representing features that are shared by objects from different clusters through marked segments placed along the appropriate branches of the tree. The marked segments corresponding to shared features between two or more objects do not enter into the calculation of the path-length distance between these objects within the tree. For example, if two stimuli are located in distinct clusters separated by a path length of $x$, and the stimuli share nonnested features represented by two marked segments (e.g. "A") along the interconnecting path with a combined distance of $y$, then the psychological distance between the stimuli is $x-y$. The distance between a similar pair of stimuli that are separated by distance $x$, but do not share nonnested features, would simply be equal to $x$. The longer the lengths of the marked segments for a stimulus pair, the more strongly they share a nonnested feature. We opted for this representational format because we expected that subjects would base their judgments of similarity of shape on several nonorthogonal factors that are not easily represented in a hierarchical or additive tree. Indeed, EXTREE solutions for the visual and haptic matrices accounted for an additional $15 \%-20 \%$ of the variance over a simple additive model.

The EXTREE solutions for the haptic and visual conditions are shown in Figures 2 and 3, respectively. The branches of the two tree structures were rotated into congruence with each other to avoid the appearance of structural differences on those occasions where only a trivial transformation of branch ordering could account for the difference. The EXTREE solutions for haptic and visual dissimilarity matrices accounted for $91.4 \%$ and $92.3 \%$ of their variance, respectively, as opposed to $77.6 \%$ and $75.5 \%$ of their variance using a basic additive tree structure without nonnested features. At first glance, there appear to be strong similarities between the two configurations that suggest that the 16 objects are perceptually equivalent for vision and touch. For example, Object Pairs $6 \mathrm{~A}-6 \mathrm{~B}, 7 \mathrm{~A}-7 \mathrm{~B}$, and $8 \mathrm{~A}-8 \mathrm{~B}$ occupy equivalent positions in the two trees, while four of the tetragons (1A, $2 \mathrm{~A}, 3 \mathrm{~A}$, and $3 \mathrm{~B}$ ) also cluster in a similar fashion. There are some discernible differences in clustering, however: (1) Within the haptic tree, Object 1B clusters with Object $5 \mathrm{~B}$ rather than with Object $3 \mathrm{~B}$, which is visually more structurally similar to it. (2) Object $3 \mathrm{~B}$, with its fundamentally rectangular shape, is haptically more similar to the two hexagonal columns, one of which (Object 6B) features narrow grooves running along its length. (3) In the visual configuration, Objects $2 \mathrm{~A}, 4 \mathrm{~A}$, and $5 \mathrm{~A}$ are located closest to those objects with similar global shape (i.e., Objects $2 \mathrm{~B}, 4 \mathrm{~B}$, and 5B, respectively), but within the haptic configuration, their closest counterparts are instead objects with less similar global shape but more similar surface properties (i.e., Pairs $4 \mathrm{~A}-5 \mathrm{~A}$ and $1 \mathrm{~A}-2 \mathrm{~A}$ have surfaces uninterrupted by local features). In general, although the two tree structures reveal a partial visual-haptic equivalence for shape, several of

Table 2

Listing of the Distinctive, Nonoverlapping Local and Global Features Corresponding to the Marked Features Contained Within the EXTREE Solutions for Experiments 1 and 2

\begin{tabular}{|c|c|c|c|}
\hline \multirow[b]{2}{*}{ Visual } & \multicolumn{3}{|c|}{ Haptic } \\
\hline & No Gloves & Thick Gloves & Splinted Gloves \\
\hline \multicolumn{4}{|c|}{ Local Features } \\
\hline \multirow{2}{*}{$\begin{array}{l}\text { Narrow angles }\left(<90^{\circ}\right) \text { between } \\
\text { surfaces }(\mathrm{A})\end{array}$} & Sharp protrusions (A) & & Sharp protrusions (A) \\
\hline & $\begin{array}{l}\text { Deep grooves (concave/ } \\
\text { convex complexity) (B) }\end{array}$ & & $\begin{array}{l}\text { Deep grooves (concave/ } \\
\text { convex complexity) (B) }\end{array}$ \\
\hline \multirow[t]{4}{*}{$\begin{array}{l}\text { Narrow grooves at intersections } \\
\text { between sides }(C)\end{array}$} & \multicolumn{3}{|c|}{$\begin{array}{l}\text { Narrow grooves at intersections } \\
\text { between sides (C) }\end{array}$} \\
\hline & & & $\begin{array}{l}\text { Rounded, multisided shapes with } \\
\text { small concave features (D) }\end{array}$ \\
\hline & \multicolumn{3}{|c|}{$\begin{array}{l}\text { Complexity (no. of sides) } \\
\text { (D) }\end{array}$} \\
\hline & \multicolumn{2}{|c|}{ Global Features } & \\
\hline $\begin{array}{l}\text { General hexagonal } \\
\text { structure }(\mathrm{E})\end{array}$ & $\begin{array}{l}\text { General hexagonal } \\
\text { structure }(\mathrm{E})\end{array}$ & $\begin{array}{l}\text { General hexagonal } \\
\text { structure }(E)\end{array}$ & $\begin{array}{l}\text { General hexagonal } \\
\text { structure }(\mathrm{E})\end{array}$ \\
\hline \multicolumn{4}{|l|}{$\begin{array}{l}\text { Hexagonal structure } \\
\quad \text { (size transformation) (F) }\end{array}$} \\
\hline \multirow[t]{3}{*}{$\begin{array}{l}\text { Rectangular/hexagonal } \\
\text { column }(\mathrm{G}, \mathrm{H})\end{array}$} & $\begin{array}{l}\text { Rectangular/hexagonal } \\
\text { column }(\mathrm{H})\end{array}$ & $\begin{array}{l}\text { Rectangular/hexagonal } \\
\text { column }(\mathrm{H})\end{array}$ & \\
\hline & $\begin{array}{l}\text { Large uninterrupted } \\
\text { surfaces (I) }\end{array}$ & & $\begin{array}{l}\text { Large uninterrupted } \\
\text { surfaces (I) }\end{array}$ \\
\hline & & $\begin{array}{l}\text { Cubical/rhomboid shape } \\
\text { (J) }\end{array}$ & $\begin{array}{l}\text { Cubical/rhomboid shape } \\
(\mathrm{D}, \mathrm{O})\end{array}$ \\
\hline
\end{tabular}




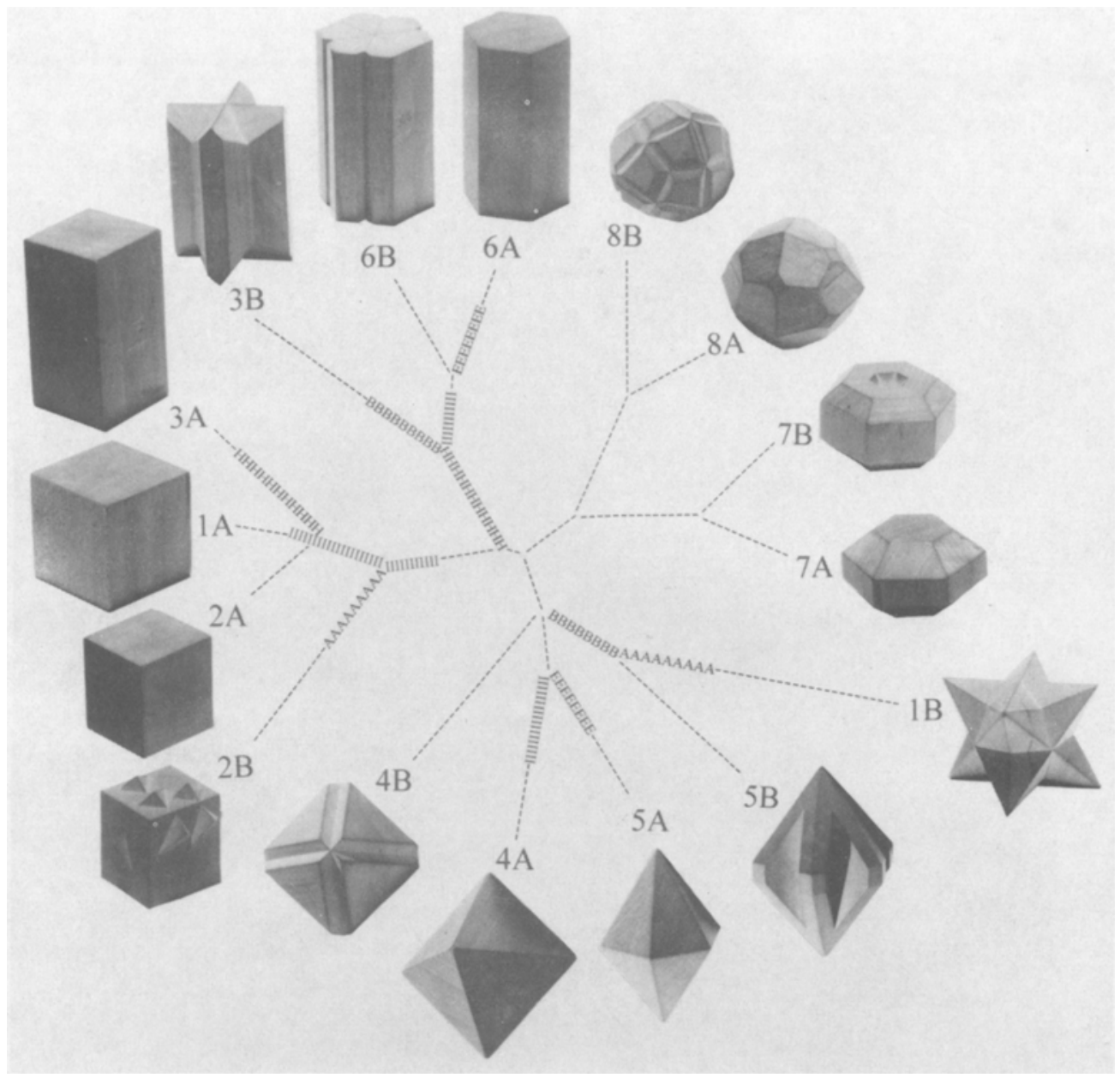

Figure 2. EXTREE solution for the first condition (haptic) of Experiment 1. Marked segments indicate nonoverlapping features.

the objects in the haptic solution cluster with objects sharing common local features, rather than with their closest global shape counterparts. This finding complements the results presented in Figure 1, showing that local object features seem to be weighted relatively more heavily by the haptic system than by the visual.

Table 2 lists the distinctive, nonoverlapping local and global features corresponding to the marked segments in Figures 2 and 3. One noticeable difference between the two modalities is that vision tends to weight those features of shape that are preserved despite differences in other local or global characteristics, while touch picks up, across clusters, primarily those features of shape that differentiate objects despite the presence of common or shared features. Both modalities, for instance, pick up features pertaining to sharp or narrow angles between surfaces (labeled Feature $A$ in Figures 2 and 3). To vision, Objects 1B, 2A, 2B, 4A, 4B, 5A, and 5B appear similar because they possess acute angles $\left(<90^{\circ}\right)$ be- tween many of their surfaces and share certain partwhole properties (e.g., Object 1B can roughly be thought of as consisting of four orthogonally interpenetrated instances of Object 5A). To touch, only Objects $1 \mathrm{~B}$ and $2 \mathrm{~B}$ are linked on the basis of their sharp protrusions, even though several other pairs of objects possess this property, as well as other common features (e.g., $1 \mathrm{~B}$ and $5 \mathrm{~A}$, as noted above). Similarly, grooves and concave indentations differentiate objects in both the visual and the haptic trees (Feature B in Figures 2 and 3). Objects 4B and $8 \mathrm{~B}$ are linked across clusters in the visual tree, but not in the haptic, because both objects have narrow grooves along their major lines of symmetry (Feature C), even though their overall shapes are quite different. Objects $1 \mathrm{~B}, 3 \mathrm{~B}$, and $5 \mathrm{~B}$ are all similar haptically, presumably because all of them have deep grooves or concave indentations at intersections between their surfaces, in spite of the much stronger overall structural similarity between Objects $1 \mathrm{~B}$ and $3 \mathrm{~B}$. 


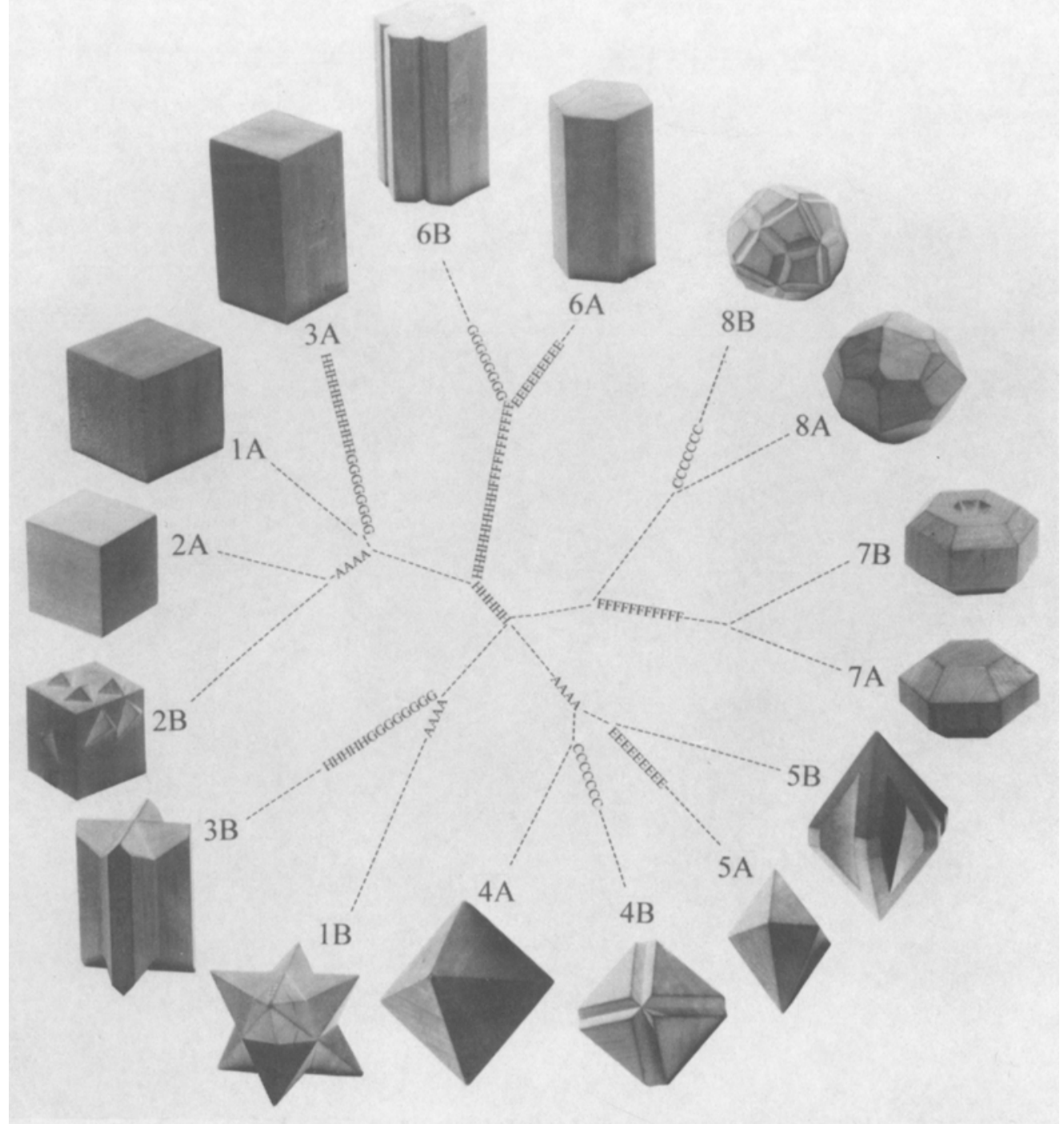

Figure 3. EXTREE solution for the second condition of Experiment 1 (visual).

Both vision and touch pick up global hexagonal and rectangular aspects of shape that cut across clusters (Features $\mathrm{E}$ and $\mathrm{F}$, respectively). Objects $5 \mathrm{~A}, 6 \mathrm{~A}$, and $6 \mathrm{~B}$ are to some extent similar because they have a general hexagonal structure. The haptic system, however, does not appear to pick up the distinctive hexagonal traits shared by Object Pairs 6A-6B and 7A-7B, which differ primarily in terms of a simple linear transformation (i.e., elongation) along one axis (Feature $\mathrm{H}$ in Figure 2).

A trait that appears particularly salient to touch alone is the presence of a relatively simple geometric part structure in objects with fundamentally different overall shapes. In Figure 2, Objects 1A, 2A, 2B, 3A, 4A, 6A, and $6 \mathrm{~B}$ share a set of features (Feature $\mathrm{I}$ ) that can best be described collectively as sets of large, uninterrupted planar surfaces. These objects possess neither a singular global shape nor a consistent set of local features, but are distinguishable from the other objects in the stimulus set by their relatively low level of spatial complexity (i.e., they are composed of a small number of geometrically simple surfaces). Because the haptic system acquires spatial information sequentially through temporally extended hand movements, it is not unexpected that objects whose shapes can be diagnosed in less time should be perceived at some level to be more similar; such objects could constitute a general class of "simple" geometric shapes that cuts across more specific categories of geometrical inclusiveness.

EXTREE solutions for the thick- and splinted-glove conditions are presented in Figures 4 and 5, respectively. Differences due to haphazard branch orientations were again minimized by rotating branches into congruence across the two trees. EXTREE solutions accounted for $91.1 \%$ and $90.7 \%$ of the variance associated with dissimilarity matrices for Conditions 3 and 4 , respectively ( $75.5 \%$ and $76.3 \%$ for a corresponding additive model). 


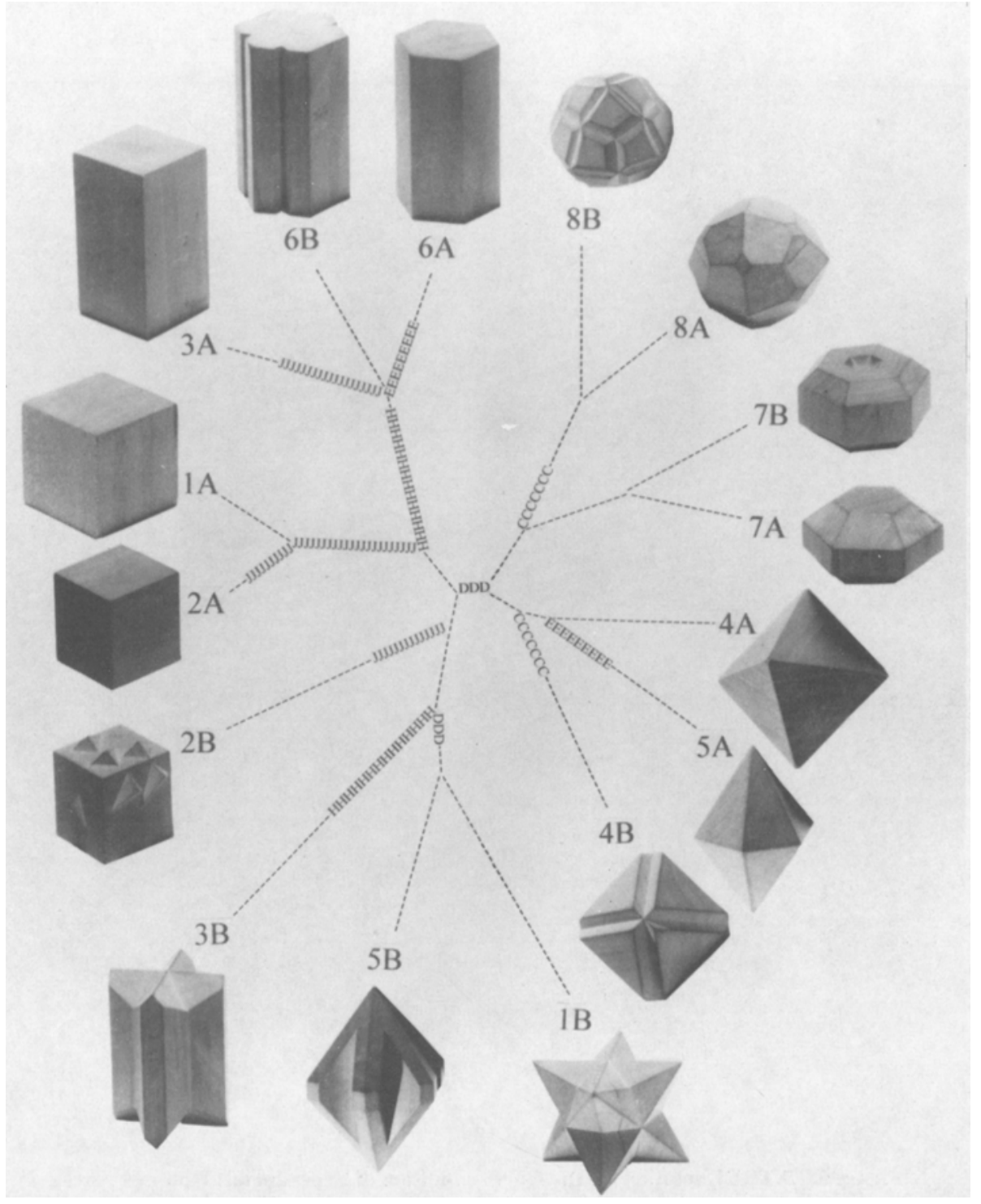

Figure 4. EXTREE solution for the third condition of Experiment 1 (thick glove).

Certain aspects of Figure 6 suggest that the spatial lowpass filtering afforded by the thick rubber gloves did direct subjects' attention somewhat more toward features of global shape than was the case for the haptic condition. Object 3B, for example, clusters more closely with Object $1 \mathrm{~B}$, as it does in the visual condition, rather than with the other columnar shapes (e.g., 3A, 6A, and 6B). Significant differences remain between Figures 3 and 4, however, implying that an artificial weighting of haptic cues toward more global aspects of shape does not necessarily bring haptic perception of shape more in line with that of the visual perception: (1) Object 5B still does not cluster with its closest global shape counterpart, Object 5A. (2) Object 2B does not cluster most closely with Object $2 \mathrm{~A}$, as it does in the visual tree, even though their overall shapes are identical. (3) Object $4 \mathrm{~A}$ is perceived as slightly more similar to Object $5 \mathrm{~A}$ than it is to Object 4B, even though both Objects $4 \mathrm{~A}$ and $4 \mathrm{~B}$ are tetragonal. Overall, the similarity ratings for the thickglove condition resemble more closely those of the haptic condition than those of the visual, which suggests that the differences between haptic and visual similarity structures cannot be reduced substantially by biasing the haptic system toward more general properties of 3-D form.

Figure 5 provides some evidence that subjects in the splinted-glove condition attended somewhat more to local features of shape, such as grooves or edges, than 


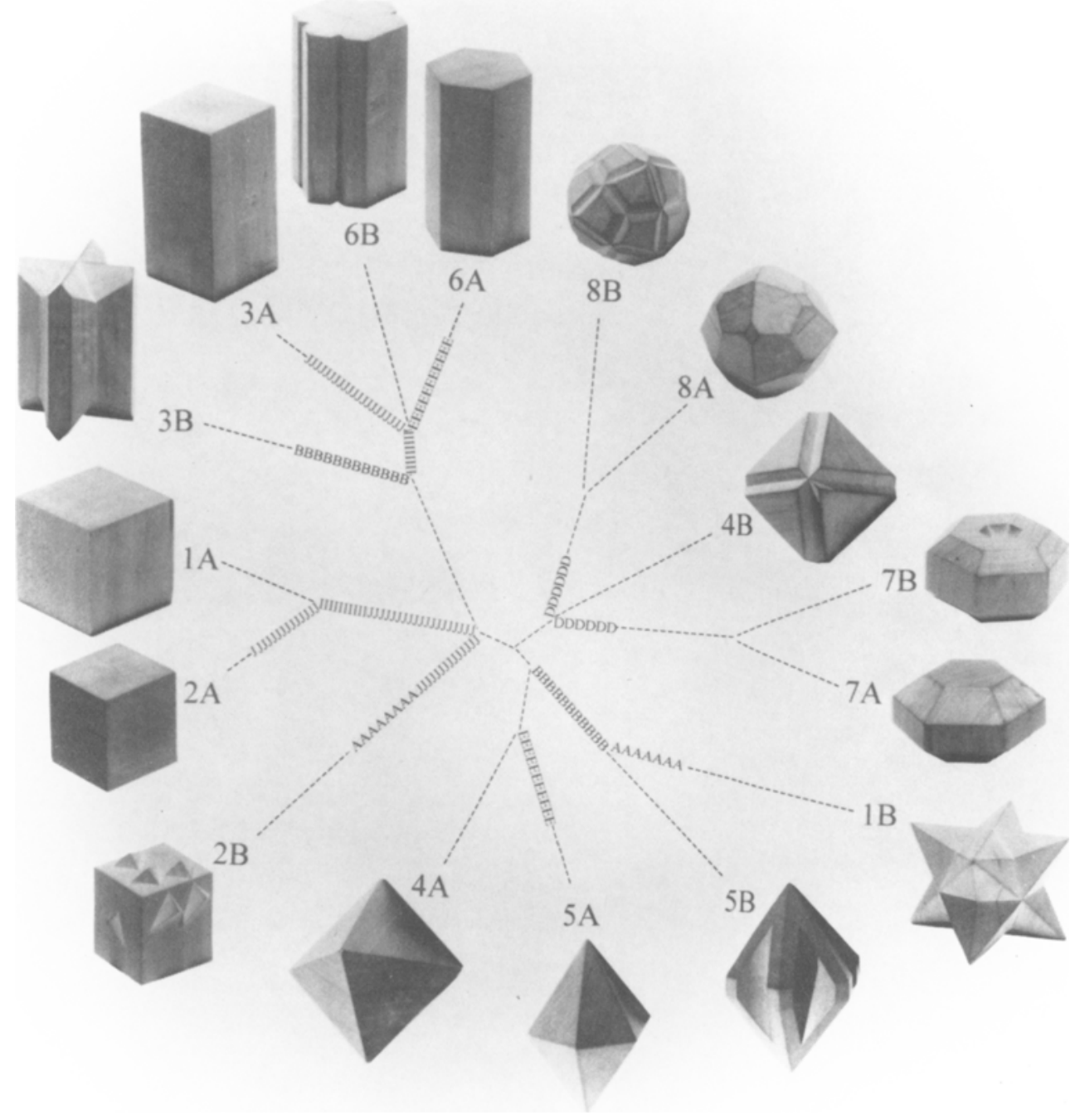

Figure 5. EXTREE solution for the fourth condition of Experiment 1 (splinted glove).

did those who explored the shapes haptically without gloves. Object 4B clusters more strongly with Object 8B, with which it shares the property of grooves running along all vertices between surfaces. Object $3 \mathrm{~A}$ groups with Objects $6 \mathrm{~A}$ and $6 \mathrm{~B}$, rather than with Object $1 \mathrm{~A}$; this is probably due to the fact that the columnar dimensions of Objects $3 \mathrm{~A}, 6 \mathrm{~A}$, and $6 \mathrm{~B}$ encourage approximately equivalent contour-following procedures, in terms of both length of time and extent. Object 2B, with its small, sharp protrusions, clusters less strongly with Object $2 \mathrm{~A}$, probably because these local features are particularly salient when the subject is engaged exclusively in contour-following.

Restricting hand movements to either enclosure or contour-following did not lead to increased prominence of certain types of spatial features diagnostic of shape, as we had expected, given the differing constraints on avail- able exploratory movements across the two conditions. As can be seen in Table 2, subjects in both the thickglove and the splinted-glove conditions picked up more or less the same features, although there are some exceptions. Both groups, for instance, weighted the general commonalties in hexagonal and cubical/rhomboid shape that cut across specific clusters, and the tree structures for both groups reflect a tendency to discriminate objects on the basis of their complexity, either in terms of their number of sides (Feature D in Figure 4) or, conversely, in terms of the presence of absence of large, uninterrupted surfaces (Feature I in Figure 5). Comparison of Figures 4 and 5 reveals that donning the splinted gloves further directed subjects' attention to the sharp protrusions of Objects $1 \mathrm{~B}$ and $2 \mathrm{~B}$ and to the deep grooves/occlusions of Objects $1 \mathrm{~B}, 3 \mathrm{~B}$, and $5 \mathrm{~B}$; on the other hand, donning rubber gloves directed attention to 


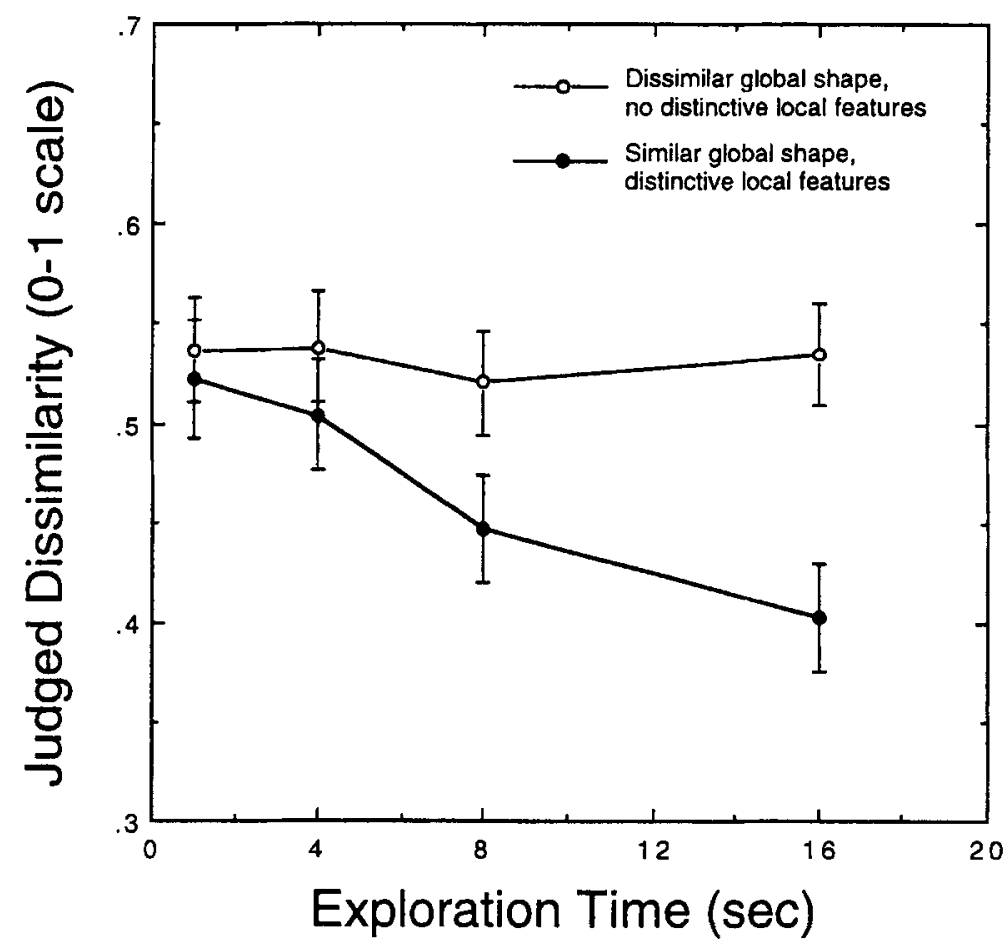

Figure 6. Average similarity ratings and standard errors, plotted as a function of exploration time for the two stimulus sets of Experiment 2: (open circles) pairs of objects with differing global features and no distinguishing local features; (filled circles) pairs of objects sharing similar global shape, but possessing distinctive local features.

such characteristics as the narrow grooves along the edges of Objects $4 \mathrm{~B}$ and $8 \mathrm{~B}$, a distinctive feature in the visual condition. Taken together, the results suggest that haptic information pertaining to local and global features of shape may be redundant in many $3-\mathrm{D}$ objects, and that therefore it may be adequately perceptible through more than a single exploratory gesture or movement. With the present stimulus set, neither enclosure nor contour-following constitutes a necessary, or even optimal, diagnostic of either local or global shape.

\section{Discussion}

The results of Experiment 1 suggest that the haptic system is relatively more attuned than the visual system to local properties as opposed to the more global aspects of object geometry. Since haptic exploration is goal driven in that hand movements are optimized to seek out particular types of spatial information, we anticipated that the placement of artificial restrictions on the availability of certain hand movements should alter the relative emphasis that the haptic system places on local versus global features of shape. In particular, by inhibiting hand movements associated with the interpretation of fine spatial structure, global aspects of shape should matter more to haptic shape perception and, consequently, haptic similarity ratings should fall better in line with visual ratings. The latter two conditions of Experi- ment 1 tested these predictions by restricting subject's hand movements to either enclosure, for diagnosing global shape, or contour-following, for perceiving spatial detail. Surprisingly, no differences in similarity ratings were found.

Although exploratory hand movements are optimized to extract specific spatial features, one can assume that information about nonoptimal features is also extracted, albeit to a lesser extent, and that with sufficient time, even a nonoptimal exploratory procedure can yield enough redundant information to create a veridical representation of 3-D shape. Because they judged only 16 objects across 240 trials, subjects in Experiment 1 may have had sufficient exposure to these shapes so that any processing differences between the two types of exploratory procedures might have been masked by overfamiliarization. On the basis of such assumptions, we surmised that changes in exploration time would affect the relative salience of local and global shape characteristics of the present stimulus set. If local features are most salient during the initial few seconds of haptic exploration, then one might expect that similarity judgments under brief exploration periods (e.g., 1-4 sec) would reflect a bias toward such features. In the same vein, if global shape properties emerge only after several seconds of exploration, then similarity ratings might shift over time toward more general shape criteria. There- 
fore, we examined in Experiment 2 the implications of varying exploration periods on the relative weighting of these different shape properties.

\section{EXPERIMENT 2}

\section{Method}

Subjects. Forty-eight subjects ( 29 women, 19 men) were paid for their participation in this portion of the study. None had participated in Experiment 1.

Stimuli. In order to test whether local features are weighted more heavily than global ones during initial exploration of an object's shape, we selected pairs of objects from among the 16 stimuli used in Experiment 1 to form two distinct sets (see below). Object pairs in Set 1 differed primarily in local shape (i.e., global shape was held roughly constant), while objects in Set 2 differed primarily in global shape.

\section{Set 1}

Object $1 \mathrm{~A} \leftrightarrow$ Object 1B Object 2A $\leftrightarrow$ Object 2B

Object $3 \mathrm{~A} \leftrightarrow$ Object 3B

Object 4A $\leftrightarrow$ Object 4B

Object $5 \mathrm{~A} \leftrightarrow$ Object $5 \mathrm{~B}$

Object 6A $\leftrightarrow$ Object 6B

Object 7A $\leftrightarrow$ Object 7B

Object $8 \mathrm{~A} \leftrightarrow$ Object $8 \mathrm{~B}$
Set 2

Object 1A $\leftrightarrow$ Object 2A

Object $1 \mathrm{~A} \leftrightarrow$ Object $4 \mathrm{~A}$

Object $2 \mathrm{~A} \leftrightarrow$ Object $5 \mathrm{~A}$

Object 3A $\leftrightarrow$ Object 6A

Object $3 \mathrm{~A} \leftrightarrow$ Object $8 \mathrm{~A}$

Object 4A $\leftrightarrow$ Object 5A

Object 6A $\leftrightarrow$ Object 7A

Object 7A $\leftrightarrow$ Object 8A
Given the shape constraints of our initial stimulus set (i.e., eight pairs of objects sharing global features but differing in terms of local features), Set 2 consisted of eight objects paired with each other in such a way that each object was presented twice within the set, whereas Set 1 consisted of 16 objects, each being presented only once. In Set 2, no objects had local features (i.e., all were objects labeled "A"; see Table 1), whereas one object in each pair in Set 1 had local features (i.e., were labeled "B"; see Table 1). A1though this presents the potential for an unbalanced design since objects labeled "B" occur one third as frequently across Sets 1 and 2 as objects labeled "A," we anticipated that the short presentation times and limited number of trials for each subject would preclude any strong biases due to differences in the frequency of presentations for the "A" and " $B$ " stimuli.

Apparatus and Procedure. Subjects compared the shapes of all 16 pairs of objects listed above once each in the course of a single 15-min, 16-trial session. On each trial, subjects were presented with one of the pairs, using the apparatus from Experiments 1. Exploration time varied randomly from trial to trial $(1,4,8$, or $16 \mathrm{sec})$. The combination of exploration time and object pairing followed a $4 \times 16$ factorial design across groups of 4 subjects in such a way that each of 4 subjects explored each pair of objects for one of the four exploration times, and each subject made an equal number of comparisons (i.e., four stimulus pairs) at each exploration time per session. Therefore, each subject did not compare each pair at all four lengths of time, but, instead, groups of 4 subjects judged all possible combinations of object pairing versus exploration time. The main purpose of this design was to limit each subject's exposure to the stimulus set as much as possible while still obtaining data for all cells of the factorial design. Trials were presented randomly within sessions.

Each trial began when the experimenter announced the amount of time the subject would have to explore the object pair (times were announced in advance so that subjects would be able to optimize their exploratory procedures for a given observation interval). The experimenter then initiated a 20 -sec countdown on the screen of an IBM-PC. Subjects were instructed to hold their hands approximately $10 \mathrm{~cm}$ above the observation table so as to prepare to grasp the pair of objects as quickly as possible. Three consecutive instructions were given to subjects: (1) a verbal hazard ("Get ready") by the experimenter $3 \mathrm{sec}$ before the beginning of the exploration period; (2) a verbal cue by the experimenter to pick up and explore the shapes ("Go"), after which time the subjects explored the shapes for the full duration allotted; and (3) a verbal cue by the experimenter to end the exploration period ("Stop"). Subjects then rated the similarity of shape using the procedure of Experiment 1 . The experimenter coordinated the verbal cues through visual icon prompts on his/her computer monitor. Pilot testing of this procedure revealed it to be superior to computer-generated visual or auditory cues, in that subjects felt more relaxed with the experimenter's prompts; they also timed the pick-up and release of objects with more consistent accuracy.

A calibration trial preceded the start of the experiment. Subjects were presented with a single pair of objects (Objects 6A and 6B) and told that this reference pair should be considered very similar (i.e., at the far left end of the similarity scale, although not necessarily at the extreme left-most position of the slider switch) and that they should make all subsequent similarity ratings with respect to this pair. The goal of this calibration trial was to anchor all subjects' judgments to a common referent. Objects $6 \mathrm{~A}$ and $6 \mathrm{~B}$ were also paired once in the experimental session, but subjects' similarity ratings for this pair were excluded from subsequent data analyses. (We did check to see, however, that subjects indeed rated this pair as highly similar, and thus were following directions. All subjects did so.)

\section{Results}

Ratings for the two sets of objects - (1) similar global shape but different local features versus (2) different global shapes lacking local features-are plotted separately in Figure 6 as a function of exploration time (each point represents the mean of 96 judgments). Separate ANOVAs on the two sets of data showed a significant effect of exploration time on relative similarity for Set 1 objects $[F(3,33)=7.99, p<.001]$, but not for those of Set $2[F(3,33)=.168, p=.92]$. Interactions between exploration time and pairing of objects were nonsignificant for both Set $1[F(21,231)=1.51, p=.07]$ and Set 2 $[F(21,231)=1.24, p=.22]$, implying that the effect of exploration time on ratings of Set 1 objects was not driven only by a subset of the eight pairs.

Overall, subjects judged pairs from Set 1 to grow increasingly similar with increases in exploration time from 1 to $8 \mathrm{sec}$ (from 8 to $16 \mathrm{sec}$, there was no significant increase in similarity rating). The same was not true for pairs from Set 2, where similarity ratings remained relatively constant across all four exploration times. This systematic change in relative similarity for Set 1 pairs over time suggests that subjects initially tend to perceive objects with similar global shape, but different local structure, to be relatively dissimilar. But with time, the relative salience of the distinctive local features decreased and subjects tended to perceive the global structural similarities to a progressively greater extent. In the case of objects with differing global shape, but with no distinguishing local features, judgments of similarity appear to have become stable after only 1 or $2 \mathrm{sec}$.

These findings seem even more intriguing in light of the fact that our informal observations showed that all subjects used enclosure exclusively for the 1 - and 4-sec exploration periods, and engaged in contour-following only during later portions of the 8-and $16-$ sec periods. If enclosure is associated primarily with the apprehension 
of global shape and contour-following with the apprehension of more local features, then one would expect that local features would tend to influence shape judgments less during the initial few seconds of haptic exploration, and that with the later application of contourfollowing, these same features would become progressively more salient. Instead, we found the opposite for those object pairs that shared similar global shape: Differences in local features tended to exert their greatest influence during early exploration, and progressively less so given longer time periods.

Taking both sets of findings together implies that touch is quite rapid at discerning global shape when there are no local or small-scale features. However, distinctive small-scale features may draw haptic attention away from more structurally relevant global information during the initial stages of exploration, until successive hand movements enact a type of temporal low-pass spatial filtering in which these local features become less salient and global ones become more perceptually prominent. Because the present experiments have shown that touch weights local features more heavily than vision, one might speculate that touch initially focuses on the local shape of objects, and only later does a sense of overall shape emerge. Vision, with its more direct apprehension of 3-D space, picks up on the global qualities of objects, followed by more careful observations of smaller surface features.

\section{GENERAL DISCUSSION}

The present experiments examined the differential emphasis that the haptic system places on local and global features of 3-D objects. Haptic processing of spatial form is distinct from that of vision in that touch does not have rapid, simultaneous access to both small- and large-scale aspects of an object, but must interpret relatively small surface areas in succession using sequences of specialized hand movements. The sequential nature of haptic exploration suggests that prominent features consisting of sharp edges, points, and contours may appear more salient, at least initially, to touch than to vision. Experiment 1 provided evidence that this is the case. Subjects in the haptic condition rated pairs of objects differing primarily in terms of the presence or absence of distinguishing local features as significantly less similar than did subjects who made the same comparisons visually, relative to the overall mean for all other pairs. Clustering solutions obtained from haptic and visual similarity matrices also reveal systematic differences between the two modalities. Objects consisting of sharp points or deep surface occlusions tend to cluster with other objects sharing these local features, rather than with their global shape counterparts, in the haptic sense.

The findings from the visual and haptic conditions of Experiment 1 would seem to support a direct apprehension model of haptic perception rather than one postulating that haptic spatial information is translated into a visual image at a relatively early stage of processing. The clustering solution for the haptic condition cannot be interpreted simply as a coarse or degraded variant of the visual configuration; rather, certain objects among the 16 stimuli cluster differently when haptic comparisons are made, and such differences stem from the relatively greater weight assigned to local shape characteristics. Such an interpretation runs counter to the findings from Conditions 3 and 4 , however, in which haptic exploration was constrained to either enclosure or contour-following by means of restrictive gloves. Subjects in the thickglove and splinted-glove conditions assigned more or less equivalent similarity ratings to most, if not all, pairs of objects, despite our efforts to bias the subjects' exploratory hand movements toward the extraction of either global shape (through enclosure) or local shape (through contour-following). This outcome seems at first glance to run counter to a considerable body of research showing that a hallmark of haptic processing is the presence of distinct exploratory procedures for perceiving different types of spatial and nonspatial cues. At the very least, it suggests that, for certain stimuli, specific hand movements may not be so specialized as previously thought, and that they may be diagnostic of a fairly broad range of haptic cues, even at suboptimal processing levels.

One possible explanation comes from the constraints of the paradigm in Experiment 1. Given that subjects explored a limited set of objects over a long period ( 240 trials over 2 nonconsecutive hours), they may have been able to construct a fairly elaborate and stable representation of each object's shape, similar to the manner in which one might build up a coherent visual image of a large object by viewing it from multiple, disparate perspectives. In this sense, the use of an exploratory gesture such as enclosure can still yield considerable information about local structure, provided that sufficient time is available for exploration. "Optimal" may refer not only to the degree of correlation between the hand movement and the type of haptic information sought but also to the efficiency with which the movement can be executed in time. It is also possible that subjects wearing thick or splinted gloves shifted their exploration strategies to compensate for restrictions in hand movement; for example, contour-following may have been directed more toward identifying global aspects of shape than would be the case under unrestricted exploration. When only a subset of exploratory movements is available in a given context, individuals may be able to shift their strategy for attending to object features so that nonoptimal features receive greater attention. Both extended stimulus exposure and shifts in exploration strategies may account for the relatively constant performance in Conditions 3 and 4 of Experiment 2.

At least one criterion that changes with time is the relative salience of local object characteristics. In Experiment 2 , the relative similarity between objects distinguished primarily by local features increased as a function of exploration time, while similarity remained 
constant for objects differing primarily in terms of global shape. These findings are not unexpected for two reasons. First, with the present stimulus set, objects with local features tend to be more complex, both in terms of their number of sides and the manner in which these sides intersect. Since hand movements can provide only a certain amount of information within a given time period, a complex object would require longer exploration periods for a complete impression of shape. Second, because each fingertip of the hand has a relatively small surface area (approximately $1-3 \mathrm{~cm}^{2}$ in adults), object features that have equivalent dimensions (e.g., edges, grooves, vertices) will tend to indent the skin to a greater extent and will be more salient (at least initially) than will features that have an area substantially larger than that of the fingertip.

An initial impression of an object's shape based on a smaller number of hand excursions will therefore be based primarily on features with areas similar to those of the fingertips. In the case of objects with large numbers of such small-scale features, such initial impressions may be quite unrepresentative of the general shape of the object if local and global features are only modestly correlated. If, on the other hand, an individual has several seconds or more to explore, several redundant excursions over an object's surface may yield a more convincing impression of overall shape. This may be due to a type of low-pass spatial filtering achieved through successive sampling of an object's contours, much the same as one would scan a large object with the eye by superimposing several visual images of an object to yield a composite image comprising the object's primary features and contours. As Krueger noted in his editor's introduction to Katz (1989):

In the impression of the ordinary object, according to Katz, movement, time, and space leave no trace of themselves; the object is precipitated as an independent entity, largely uncontaminated by its journey through tactual time and space. When a person glides his or her hand over a motionless object, say the corner of a chair, the hand touches different and constantly changing parts of the corner, Katz said, yet the corner persists just as steadfast in tactual space as do objects in visual space when the eye is moved. (p. 15)

The notion of touch as a modality that weights features differentially over time based on a continuously evolving impression of local and global shape is one that merits further attention.

\section{REFERENCES}

Balakrishnan, J. D., KLATZKy, R. L., LoOmis, J. M., \& Lederman, S. J. (1989). Length distortion of temporally extended visual dis- plays: Similarity to haptic spatial perception. Perception \& Psychophysics, 46, 387-394.

Brown, D. R., \& Brumaghim, S. H. (1968). Perceptual equivalence, pattern perception, and multidimensional methods. Perception \& Psychophysics, 4, 253-256.

Brown, D. R., \& OWEN, D. H. (1967). The metrics of visual form: Methodological dyspepsia. Psychological Bulletin, 68, 243-259.

Corter, J. E., \& TVErsky, A. (1986). Extended similarity trees. Psychometrika, 51, 429-451.

Davidson, P. W., AbBott, S., \& Gershenfeld, J. (1974). Influence of exploration time on haptic and visual matching of complex shape. Perception \& Psychophysics, 15, 539-543.

GARBIN, C. P. (1990). Visual-touch equivalence for shape information in children and adults. Perception \& Psychophysics, 48, 271-279.

Garbin, C. P., \& BERNSTEIN, I. H. (1984). Visual and haptic perception of three-dimensional solid forms. Perception \& Psychophysics, 36, 104-110.

GiBson, J. J. (1966). The senses considered as perceptual systems. Boston: Houghton Mifflin.

Heller, M. A., Calcaterra, J. A., Burson, L. L., \& Tyler, L. A. (1996). Tactual picture identification by blind and sighted people: Effects of providing categorical information. Perception \& Psychophysics, 58, 310-323.

KaTZ, D. (1989). The world of touch (L. E. Krueger, Ed, and Trans.). Hillsdale, NJ: Erlbaum. (Original work published 1925)

Klatzky, R. L., \& Lederman, S. J. (1993). Spatial and nonspatial avenues to object recognition by the human haptic system. In N. Eilan, R. McCarthy, \& B. Brewer (Eds.), Spatial representation (pp. 191205). Cambridge, MA: Blackwell.

KLATZKY, R. L., \& LEDERMan, S. J. (1995). Identifying objects from a haptic glance. Perception \& Psychophysics, 57, 1111-1123.

Klatzky, R. L., Lederman, S. J., \& MetzGer, V. A. (1985). Identifying objects by touch: An "expert system." Perception \& Psychophysics, 37, 299-302.

LEDERMAN, S. J., \& KLATZKY, R. L. (1987). Hand movements: A window into haptic object recognition. Cognitive Psychology, 19, 342368.

Lederman, S. J., \& KLATZKY, R. L. (1990). Haptic classification of common objects: Knowledge-driven exploration. Cognitive $P_{\text {sychol- }}$ ogy, 22, 421-459.

Lederman, S. J., Klatzky, R. L., Chataway, C., \& Summers, C. D. (1990). Visual mediation and the haptic recognition of two-dimensional pictures of common objects. Perception \& Psychophysics, 47, 54-64.

MAGEE, L. D., \& KENNEDY, J. M. (1980). Exploring pictures tactually. Nature, 278, 287-288.

MiLLAR, S. (1991). Reversed lag in the recognition and production of tactual drawings: Theoretical implications for haptic coding. In M. A. Heller \& W. Schiff (Eds.), The psychology of touch (pp. 301-325). Hillsdale, NJ: Erlbaum.

OWEN, D. H., \& Brown, D. R. (1970). Visual and tactual form discrimination: Psychophysical comparison within and between modalities. Perception \& Psychophysics, 7, 302-306.

Rosch, E. H., Mervis, C. B., Gray, W. D., Johnson, D. M., \& BoyesBraEM, P. (1976). Basic objects in natural categories. Cognitive Psychology, 8, 382-439.

Sattath, S., \& Tversky, A. (1977). Additive similarity trees. Psychometrika, 42, 219-345.

TVERSKY, B., \& HeMENWAY, K. (1984). Objects, parts, and categories. Journal of Experimental Psychology: General, 113, 169-193.
(Manuscript received May 1, 1997; revision accepted for publication March 31,1998 .) 